\title{
MULTILEVEL INTERACTIONS BETWEEN THE SYMPATHETIC AND PARASYMPATHETIC NERVOUS SYSTEMS: A MINIREVIEW
}

\author{
Ondicova K, Mravec B \\ Institute of Pathophysiology, Faculty of Medicine, Comenius University and Institute of Experimental Endocrinology, \\ Slovak Academy of Sciences, Bratislava, Slovakia
}

\begin{abstract}
In order to allow precise regulation of bodily functions, the activity of the autonomic nervous system must be precisely regulated. The traditional model concerning the regulation of norepinephrine and acetylcholine release in target tissues suggests that the activities of the efferent arms of the autonomic nervous system are more or less independent of each other. However, plenty of experimental and clinical studies have demonstrated the presence of multiple interactions between the sympathetic and parasympathetic nervous system that are mediated through several pathways and mechanisms at both central and peripheral levels of the neuraxis. Interactions within the central nervous system are mediated predominantly by neurons within the nucleus of the solitary tract and paraventricular hypothalamic nucleus. Peripheral interactions are based on the morphological-functional organization of the sympathetic and parasympathetic pathways at the levels of the sympathetic prevertebral ganglia or neuroeffector connections. Furthermore, evidence suggests that neuroeffector connections may be realized at the axo-axonal, presynaptic, postsynaptic, and post-receptor levels.

Alterations in interactions between the sympathetic and parasympathetic nervous system can lead to unbalanced autonomic activities, which may influence the development of various disorders, including cardiovascular, inflammatory, metabolic, neurological, and psychiatric diseases.

The aim of this article is to illustrate the complexity of interaction between the sympathetic and parasympathetic nervous systems and to describe the role of these interactions in the heart, adrenal medulla, and vagal trunk.
\end{abstract}

Key words: Autonomic ganglia - Paracrine modulation - Parasympathetic - Postsynaptic Presynaptic - Sympathetic

\section{Introduction}

Almost all bodily functions are regulated by the autonomic nervous system (ANS). Precise regulation of organ and tissue functions by the ANS requires that activity of the efferent arms of the ANS - the sympathetic and parasympathetic nerves - must be finely tuned. Therefore, the sympathetic and parasympathetic nervous systems do not work independently of each other but their activity is a result of multiple interactions at various levels of the neuraxis and at the level of the peripheral effector cells. These interactions extend to the processing of information by the ANS and preciosity of the regulation of effector organ activity (JÄNIG, 2006).

Whereas interactions between the sympathetic and parasympathetic nervous systems are necessary for the precise regulation of bodily functions during physiological situations, the importance of these interactions are 
accentuated during pathological conditions (e.g., heart failure, inflammation, stroke - Micieli and CAVALLINI 2008; Olshansky et al. 2008; Sternberg 2006).

\section{Central interactions}

Interactions between the sympathetic and parasympathetic nervous systems at the level of the central nervous system may be realized through several pathways and mechanisms (Fig. 1). These interactions may result from the processing of information transmitted by neuronal and humoral pathways to the nucleus of the solitary tract (NTS) or paraventricular hypothalamic nucleus (PVN). These two prominent structures in turn regulate the activity of the sympathetic and parasympathetic nervous systems. This regulation has features similar to a reflex mechanism (e.g. baroreflex or chemoreflex - ANDRESEN et al. 2004). The basis for these interactions represents the transmission of information related to changes in the viscera and environment of an organism via afferent pathways that in turn regulate the activity of central parts of the ANS and, consequently, the activity of the sympathetic and parasympathetic nerves. This processing of information constitutes the basis for vago-vagal, vago-sympathoadrenal, and somato-autonomic reflexes (KeRMAN and YATES 1999; Osaka et al. 2002; Powley and Phillips 2002).

Nucleus of the solitary tract. The regulation of blood pressure (baroreflex) is one example of the above-mentioned mechanisms. Information related to the blood pressure is transmitted from baroreceptors localized in the aortic arch and carotid sinus to the dorsomedial part of NTS in the medulla. Neurons of the NTS project directly or indirectly to structures of the medulla that regulate the activity of the sympathetic and parasympathetic preganglionic neurons. The activity of the parasympathetic cardioinhibitory preganglionic neurons localized mainly in the ventrolateral part of nucleus ambiguus (NA) is increased probably via direct excitatory pathways originating from the NTS. Sympathoexcitatory neurons that innervate sympathetic preganglionic neurons in the intermediolateral cell column are localized within the rostral part of the ventrolateral medulla (RVLM). Reflex control of RVLM neuron activity is mediated by an inhibitory monosynaptic pathway of neurons originating from the caudal part of the ventrolateral medulla (CVLM). Activity of the CVLM is regulated by excitatory projections from NTS neurons. Reflex circuits of the medulla constituting the baroreflex enable independent control of sympathetic and parasympathetic nerve activities. The sympathetic part of the efferent arm of the baroreflex originates in the RVLM whereas the parasympathetic part originates in the NA. The activities of these individual arms may be modulated at the level of the medulla via interactions between neurons of the RVLM and the NA, which probably interact reciprocally via functional connections. The following observations provide proof of these interactions: 1. spontaneous activity of RVLM neurons is inhibited when the vagus nerve is cut in the cervical region and the proximal end is electrically stimulated; 2. microapplication of lidocaine into the NA increases blood pressure without changing heart rate; 3 . electrolytic lesion of the NA facilitates the hypertension induced by deafferentation of sinoaortic baroreceptors (McKitrick and Calaresu 1996). Besides the functional connections between the NA and the RVLM, similar interconnections may also exist between $\mathrm{C} 1$ adrenalinergic neurons of the RVLM and the dorsal motor nucleus of the vagus (DMV - CARD et al. 2006).

Paraventricular hypothalamic nucleus. Regulation of ANS activity by the paraventricular hypothalamic neurons represents another example of central interactions. PVN neurons receive and process four main types of inputs: viscerosensitive, humoral, limbic, and intrahypothalamic. Viscerosensitive signals originate in visceral receptors, nociceptors, and thermoreceptors and are transmitted via spinal afferent pathways to the dorsal horns of the spinal cord and via vagal viscerosensitive afferent pathways to the NTS. This information is consequently transmitted to the PVN via direct pathways from the dorsal horns of the spinal cord and from the NTS or via indirect pathways after the information is processed in the parabrachial nucleus or A1/C1 cell groups of the medulla. Humoral signals are transmitted to the PVN by several mechanisms. Some signals (e.g., molecules of circulating steroids, plasma glucose concentration, osmolality) may influence the PVN directly or through local interneurons, whereas other signals (e.g., circulating angiotensin II) act via receptors in circumventricular organs (e.g., subfornical organ) which lack a blood-brain-barrier. Circulating cytokines (e.g., IL-1) may activate vagal viscerosensitive pathways and consequently influence the activity of the PVN via NTS neurons or A1/C1 cell groups. Limbic inputs from the orbitomedial prefrontal cortex and amygdala that are related to the emotional reaction to stressors from the external environment are transmitted to the PVN via neurons of the bed nucleus stria terminalis and the dorsomedial hypothalamic nu- 
cleus. The PVN also receives many intrahypothalamic inputs, not only from the dorsomedial nucleus but also from the suprachiasmatic nucleus, that represent key structures regulating circadian control of autonomic and endocrine functions, and from the arcuate nucleus and perifornical areas that are involved in the regulation of food intake and metabolism (BENARroch 2005). The modulation of PVN neuron activity by suprachiasmatic nucleus neurons enables circadian regulation of plasma glucose levels; two groups of PVN neurons participate in this regulation: sympathetic premotor neurons that regulate glucogenesis in the liver and parasympathetic premotor neurons that regulate the release of insulin from the pancreas (KALSBEEK et al. 2008).

The PVN contains several neurochemically distinct groups of neurons that send axons to autonomic nuclei in the brainstem and spinal cord (e.g. RVLM, NTS, DMV, sympathetic preganglionic neurons, sacral parasympathetic preganglionic neurons) and enable the PVN to specifically control the activity of sympathetic and parasympathetic nerves. Sympathetic and parasympathetic premotor neurons of the PVN are anatomically distinct. Neurons innervating motor nuclei of the vagus nerve are localized in the ventromedial and lateral parvocellular regions of the PVN; neurons innervating spinal preganglionic neurons are localized in the dorsal and caudal regions of the PVN (Palkovits 1999). The neuroanatomical and functional features the PVN mentioned above appear analogous with the NTS. Both the PVN and the NTS participate in the reflex regulation of ANS activity. For example, these regulations are involved in the modulation of cardiovascular system activity during physiological (e.g., sympathoexcitatory reaction during stress) and pathological situations (BENARROCH 2005). Although the PVN is a key structure important for integrating and modulating the activities of an organism's basic regulatory systems (the nervous, endocrine, and immune systems), interactions between the groups of PVN neurons that selectively regulate activity of the sympathetic and parasympathetic nerves are not known in detail.

Autonomic preganglionic neurons. Studies of the central interactions between structures regulating the activity of the sympathetic and parasympathetic systems have shown that application of a retrograde tracer to the sympathetically denervated pancreas labels sympathetic preganglionic neurons in the intermediolateral cell column of the spinal cord. Similarly, application of a retrograde tracer to a parasympathetically denervated pancreas labels DMV neurons. These data indicate the

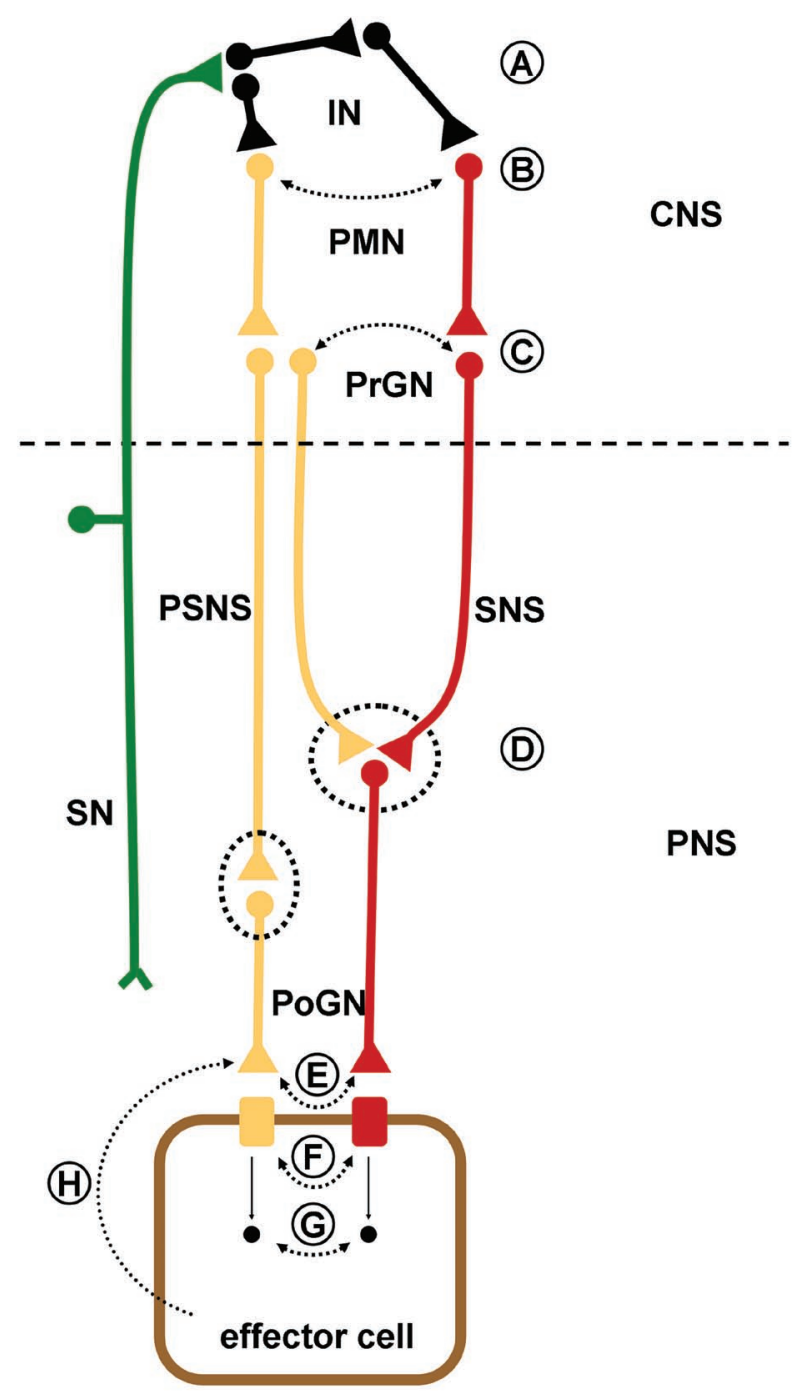

Fig. 1 Schematic drawing of the interactions between the sympathetic (SNS) and parasympathetic (PSNS) nervous systems at the levels of the central (CNS) and peripheral (PNS) nervous systems. Shown are the interactions (A) among the central structures processing sensitive information, (B) among autonomic premotor neurons, $(\mathrm{C})$ among autonomic preganglionic neurons, (D) at the level of the sympathetic prevertebral ganglia, and (E) at the presynaptic, (F) postsynaptic, and (G) intracellular levels. (H) Activity of the autonomic nervous system is modulated by compounds released in a paracrine manner by the target tissues. IN, interneurons; PMN, autonomic premotor neurons; PoGN, postganglionic neurons; PrGN, preganglionic neurons; $\mathrm{SN}$, sensory neurons.

existence of neuroanatomical interconnections between the sympathetic and parasympathetic preganglionic neurons and constitute the basis for functional inter- 
actions between the sympathetic and parasympathetic systems, at the central level (Buijs et al. 2001).

\section{Peripheral interactions}

Peripheral interactions are based on the morphological-functional organization of the sympathetic and parasympathetic pathways. These interactions may be realized at the levels of the sympathetic prevertebral ganglia and neuroeffector connections. Interactions at the level of the neuroeffector connections may be effected at the axo-axonal, presynaptic, postsynaptic, and postreceptor levels (Fig. 1). Neuroeffector interactions have been studied in detail, especially in the heart (LANGER and Hicks 1984; Myslivecek and Trojan 2003).

Sympathetic prevertebral ganglia. The interconnections between sympathetic and parasympathetic fibers found in some autonomic ganglia create the basis for peripheral interactions between sympathetic and parasympathetic systems. One example is the innervation of sympathetic prevertebral ganglia by preganglionic neurons of the vagus nerve (BERTHOUd and Powley 1993).

Interactions between the vagal preganglionic nerves and sympathetic postganglionic neurons play an important role in the effects of the cholinergic antiinflammatory pathways. Experimental studies have shown that efferent pathways of the vagus nerve exert a potent anti-inflammatory effect through acetylcholine release, which then binds to the $\alpha 7$ subtype of nicotinic receptors on immune cells. As a result, the term 'cholinergic anti-inflammatory pathway' was introduced (TRACEY 2007). The spleen is thought to play a key role in the vagal anti-inflammatory pathway (Huston et al. 2006). Although some laboratories have shown a direct vagal innervation of the spleen (Buijs et al. 2008), parasympathetic innervation of the spleen remains questionable because nerve endings in close proximity to spleen macrophages are catecholaminergic instead of cholinergic. Therefore, the acetylcholine released from efferent vagal fibers may not directly influence splenic macrophages functions. Acetylcholine released from vagal preganglionic neurons may bind to the $\alpha 7$ subtype of nicotinic receptors on sympathetic postganglionic cells localized within the coeliac and superior mesenteric ganglia and consequently, through the axons of these sympathetic postganglionic neurons, this event may modulate the activity of the splenic immune cells. Therefore, it is suggested that the vagus nerve may, through the sympathetic prevertebral ganglia, modulate the activity of the adrenergic nerves innervating the spleen. Catecholamines released from nerve endings of the splanchnic nerve stimulate the adrenergic receptors of spleen macrophages and inhibit their activity (Rosas-BALlina et al. 2008; SCHAFER et al. 1998; VAN Der Zanden et al. 2009).

Axo-axonal interactions. Sympathetic nerves are closely apposed to, and form functional synapses with, parasympathetic axons. These prejunctional interactions between sympathetic and parasympathetic axons play an important role in the regulation of many effector organ activities. Functional associations between sympathetic and parasympathetic nerve fibers imply an intimate structural relationship between these two nerve systems. The basis for this relationship stems from the close juxtaposition of the sympathetic and parasympathetic fibers within a common Schwann cell sheath in the ground plexuses of various target organs, and from the direct axo-axonal contact observed between preterminal axons and varicosities (Hasan and Smith 2000).

Presynaptic interactions. Presynaptic interactions realized through presynaptically localized heteroreceptors for acetylcholine, norepinephrine, and neuropeptide $Y$ enable reciprocal inhibition of neurotransmitter release from nerve endings of the sympathetic and parasympathetic postganglionic neurons. Acetylcholine, through binding to presynaptic muscarinic receptors localized on sympathetic nerve endings, decreases the release of norepinephrine from these nerves (BоEнм and Kubista 2002; Kubista and Воeнм 2006; Langer 2008). On the contrary, norepinephrine released from activated sympathetic nerves acts through the presynaptic $\alpha$-adrenergic receptors localized on the endings of parasympathetic postganglionic neurons and inhibits the release of acetylcholine. Similarly, neuropeptide $Y$ released from sympathetic nerve endings inhibits the effects of the vagus nerve on heart activity; on the other hand, vagal stimulation inhibits the release of neuropeptide Y from sympathetic nerves (ELGHOZI and JuLIEN 2007; Herring et al. 2008; LANGER 1997; WetZeL and Brown 1985). A role for other autonomic co-transmitters (e.g. ATP, galanin) in the presynaptic modulation of autonomic nerve activities cannot be excluded. The above-mentioned presynaptic interactions between sympathetic and parasympathetic nerve endings are especially important during pathological situations, such as heart failure (AzEvedo and PARKer 1999).

Postsynaptic interactions. Postsynaptic interactions are present at the level of the effector cell membranes and at the level of the postsynaptic receptors, through 
interactions between intracytoplasmic signaling cascades activated by the binding of catecholamines and acetylcholine or their co-transmitters to the receptors of the effector cells. One example of these types of interactions is modulation of the activity of a sinoatrial node of the heart by sympathetic and parasympathetic nervous systems, with the effects of acetylcholine predominating over the effects of norepinephrine (observed with isolated rat atria). Acetylcholine, after binding to muscarinic receptors of the effector cells, activates a cascade of intracytoplasmic processes that inhibit the increase of cAMP normally induced when the $\beta$-adrenergic receptors of these cells are occupied (ELGHOZI and JULIEN 2007).

The above-mentioned presynaptic and postsynaptic interactions between the sympathetic nerves and the vagus nerve suggest that reciprocal inhibitory effects exist. However, it is not possible to explain the depicted observation simply on the basis of neurotransmitter interactions - a more complex view is necessary. As an example, sympathetic co-stimulation increased the effect of vagus nerve stimulation on heart activity; this phenomenon was named "accented antagonism" and indicates that the effect of sympathetic and vagal stimulation on heart rate is complex and cannot be explained by simply adding the individual effects of the sympathetic and parasympathetic divisions of the ANS on the effector cells (ELghozI and Julien 2007).

Paracrine effects. Activity of the autonomic nerves may be modulated in a paracrine manner. For example, natriuretic peptides released from the myocardium itself may act on autonomic neurons innervating the heart. It has been suggested that atrial natriuretic peptides can augment the bradycardia that occurs in response to efferent vagal nerve stimulation in vivo, due to adrenergic-cholinergic cross-talk via $\alpha_{1}$-adrenergic receptors on parasympathetic ganglia (Herring and PATERSON 2009).

\section{Examples of peripheral interactions}

The most prominent peripheral interactions between the sympathetic and parasympathetic nervous systems are found in the sympathetic prevertebral ganglia (see above), intracardiac nervous system, adrenal medulla, and the vagal trunk.

Intracardiac nervous system. The intrinsic cardiac nervous system, constituted by interconnected heart ganglia, plays an important role in the regulation of heart functions (Kukanova and Mravec, 2006). Heart gan- glia contain a heterogeneous population of sympathetic, parasympathetic, and afferent neurons that are mutually interconnected. Interactions between sympathetic and parasympathetic neurons of the heart ganglia participate in the modulation of heart rate (RANDALL et al. 2003).

Adrenal medulla. Published data suggest that the vagus nerve may influence parasympathetic innervation of the adrenal medulla. Retrograde labeling studies in rats and guinea pigs demonstrate that vagal sensory ganglia and neuronal bodies are located in the dorsal motor nucleus of the vagus (Coupland et al. 1989). It was reported that vagotomy causes atrophy of chromaffin cells in both mammals and birds (PILo et al. 1984). Moreover, stimulation of the vagus nerve increases the activity of nerve fibers innervating adrenal medulla cells (NiIJIMA 1992), which may be a consequence of the interpolation of vagal preganglionic fibers on sympathetic postganglionic fibers in the sympathetic prevertebral ganglia (for details see above).

In addition, epinephrine released from the adrenal medulla may increase the activity of afferent vagal fibers, and these fibers might in turn influence the activity of sympathetic nerves and thereby modulate the release of epinephrine (MraVEC and Hulin 2006). This modulatory effect of the effector hormones of the adrenomedullar hormonal component of the sympathoadrenal system on vagus nerve activity may represent a highly complex form of peripheral interaction between the sympathetic and parasympathetic nervous systems.

Vagal trunk. The existence of sympathetic nerve fibers within the vagus nerve represents another example of peripheral interaction between the sympathetic and parasympathetic divisions of the ANS. These fibers must be distinguished from vagal catecholaminergic fibers that originate in the DMV. Sympathetic fibers running in the vagus nerve exhibit mainly noradrenergic phenotypes; therefore, parasympathetic catecholaminergic fibers are probably dopaminergic (YANG et al. 1999).

The presence of sympathetic fibers within the vagus nerve has been described in several animal species (e.g., rat, cat, dog, and human). In cats and dogs, the sympathetic and parasympathetic trunks are combined into a common vagosympathetic trunk. The presence of sympathetic fibers within the vagus nerve may represent a common phenomenon in the ANS. However, the functional significance of the coexistence of sympathetic and parasympathetic fibers remains unclear. This organization may enable co-activation of neurons of the autonomic ganglia and effector cells by sympathetic and parasympathetic fibers (KAWAGISHI et al. 2008). 


\section{Conclusions}

The traditional model of the regulation of norepinephrine and acetylcholine release in target tissues suggests that sympathetic and parasympathetic nerve activities are driven solely via brainstem integration of various reflex afferent inputs. This model suggests that the activities of the efferent arms of the ANS are more or less independent of each other. However, this view must be modified in light of the discovery of bidirectional interactions between sympathetic and parasympathetic efferent pathways at various levels of the neuraxis and at the level of the target organs.

Multilevel interactions between the sympathetic and parasympathetic nervous systems enable the ANS to modulate the activity of an organism with an adequate level of precision. Alterations in these interactions can lead to unbalanced sympathetic and parasympathetic activities which may influence the development of various diseases, including cardiovascular, inflammatory, metabolic, neurological, and psychiatric diseases.

\section{Acknowledgments}

This work was supported by the Slovak Research and Development Agency (contract no. APVV-0045-06), VEGA grants (1/4312/07, 2/0010/09, and 1/0258/10), and a European Regional Development Fund Research and Development grant (no. NFP26240120024).

\section{References}

Andresen Mc, Doyle Mw, Bailey Tw, Jin Yh: Differentiation of autonomic reflex control begins with cellular mechanisms at the first synapse within the nucleus tractus solitarius. Braz J Med Biol Res 37, 549-558, 2004. doi:10.1590/ S0100-879X2004000400012

Azevedo Er, PARKer Jd: Parasympathetic control of cardiac sympathetic activity: normal ventricular function versus congestive heart failure. Circulation 100, 274-279, 1999.

Benarroch Ee: Paraventricular nucleus, stress response, and cardiovascular disease. Clin Auton Res 15, 254-263, 2005. doi:10.1007/s10286-005-0290-7

Berthoud Hr, Powley TL: Characterization of vagal innervation to the rat celiac, suprarenal and mesenteric ganglia. J Auton Nerv Syst 42, 153-169, 1993. doi:10.1016/0165-1838(93)90046-W

Boenm S, Kubista H: Fine tuning of sympathetic transmitter release via ionotropic and metabotropic presynaptic receptors. Pharmacol Rev 54, 43-99, 2002. doi:10.1124/pr.54.1.43

Buijs Rm, Chun SJ, Nijuma A, Romijn HJ, Nagai K: Parasympathetic and sympathetic control of the pancreas: a role for the suprachiasmatic nucleus and other hypothalamic centers that are involved in the regulation of food intake. J Comp Neurol 431, 405-423, 2001. doi:10.1002/1096-9861(20010319)431:4<405::AID-CNE1079>3.0.CO;2-D

Buijs Rm, Van Der Vliet J, Garidou Ml, Huitinga I, Escobar C: Spleen vagal denervation inhibits the production of antibodies to circulating antigens. PLoS ONE 3, e3152, 2008. doi:10.1371/journal.pone.0003152

Card Jp, Sved Jc, Craig B, Raizada M, Vazquez J, Sved AF: Efferent projections of rat rostroventrolateral medulla C1 catecholamine neurons: Implications for the central control of cardiovascular regulation. J Comp Neurol 499, 840-859, 2006. doi:10.1002/cne.21140

Coupland Re, Parker Tl, Kesse WK, Mohamed Aa: The innervation of the adrenal gland. III. Vagal innervation. J Anat 163, 173-181, 1989.

Elghozi Ju, Julien C: Sympathetic control of short-term heart rate variability and its pharmacological modulation. Fundam Clin Pharmacol 21, 337-347, 2007. doi:10.1111/j.1472-8206.2007.00502.x

HASAN W, SMITH PG: Nerve growth factor expression in parasympathetic neurons: regulation by sympathetic innervation. Eur J Neurosci 12, 4391-4397, 2000. doi:10.1046/j.0953-816X.2000.01353.x

Herring N, Lokale Mn, Danson Ej, Heaton Da, Paterson Dj: Neuropeptide Y reduces acetylcholine release and vagal bradycardia via a Y2 receptor-mediated, protein kinase C-dependent pathway. J Mol Cell Cardiol 44, 477-485, 2008. doi:10.1016/j.yjmcc.2007.10.001

Herring N, PATERSON DJ: Neuromodulators of peripheral cardiac sympatho-vagal balance. Exp Physiol 94, 46-53, 2009. doi:10.1113/expphysiol.2008.044776

Huston Jm, Ochani M, Rosas-ballina M, Liao H, Ochani K, Pavlov Va, Gallowitsch-puerta M, Ashok M, Czura CJ, Foxwell B, Tracey KJ, Ulloa L: Splenectomy inactivates the cholinergic antiinflammatory pathway during lethal endotoxemia and polymicrobial sepsis. J Exp Med 203, 1623-1628, 2006. doi:10.1084/jem.20052362

JÄNIG W: The integrative action of the autonomic nervous system. Neurobiology of homeostasis. Cambridge: Cambridge University Press, Cambridge 2006. 
Kalsbeek A, Foppen E, Schalij I, Van Heijningen C, Van Der Vliet J, Fliers E, Buijs Rm: Circadian control of the daily plasma glucose rhythm: an interplay of GABA and glutamate. PLoS ONE 3, e3194, 2008. doi:10.1371/journal. pone.0003194

Kawagishi K, Fukushima N, Yokouchi K, Sumitomo N, Kakegawa A, Moriizumi T: Tyrosine hydroxylase-immunoreactive fibers in the human vagus nerve. J Clin Neurosci 15, 1023-1026, 2008. doi:10.1016/i.jocn.2007.08.032

Kerman Ia, Yates BJ: Patterning of somatosympathetic reflexes. Am J Physiol 277, R716-724, 1999.

Kubista H, Bоенм S: Molecular mechanisms underlying the modulation of exocytotic noradrenaline release via presynaptic receptors. Pharmacol Ther 112, 213-242, 2006. doi:10.1016/i.pharmthera.2006.04.005

Kukanova B, Mravec B: Complex intracardiac nervous system. Bratisl Lek Listy 107, 45-51, 2006.

LANGER Sz: 25 years since the discovery of presynaptic receptors: present knowledge and future perspectives. Trends Pharmacol Sci 18, 95-99, 1997. doi:10.1016/S0165-6147(96)01034-6

LANGER Sz: Presynaptic autoreceptors regulating transmitter release. Neurochem Int 52, 26-30, 2008. doi:10.1016/ j.neuint.2007.04.031

Langer Sz, Hicks PE: Physiology of the sympathetic nerve ending. Br J Anaesth 56, 689-700, 1984. doi:10.1093/bja/ $\underline{56.7 .689}$

MCKitrick DJ, CAlaresu Fr: Nucleus ambiguus inhibits activity of cardiovascular units in RVLM. Brain Res 742, 203-210, 1996. doi:10.1016/S0006-8993(96)00971-7

Micieli G, Cavallini A: The autonomic nervous system and ischemic stroke: a reciprocal interdependence. Clin Auton Res 18, 308-317, 2008. doi:10.1007/s10286-008-0495-7

Mravec B, Hulin I: Does vagus nerve constitute a self-organization complexity or a „hidden network“? Bratisl Lek Listy 107, 3-8, 2006.

Myslivecek J, Trojan S: Regulation of adrenoceptors and muscarinic receptors in the heart. Gen Physiol Biophys 22, 3-14, 2003.

NiıjımA A: Electrophysiological study on the vagal innervation of the adrenal gland in the rat. J Auton Nerv Syst 41, 87-92, 1992. doi:10.1016/0165-1838(92)90130-9

Olshansky B, Sabbah Hn, Hauptman PJ, Colucci Ws: Parasympathetic nervous system and heart failure: pathophysiology and potential implications for therapy. Circulation 118, 863-871, 2008. doi:10.1161/CIRCULATIONAHA.107.760405

Osaka T, Kоваyashi A, Inoue S: Vago-sympathoadrenal reflex in thermogenesis induced by osmotic stimulation of the intestines in the rat. J Physiol 540, 665-671, 2002. doi:10.1113/jphysiol.2001.013475

Palkovits M: Interconnections between the neuroendocrine hypothalamus and the central autonomic system. Geoffrey Harris Memorial Lecture, Kitakyushu, Japan, October 1998. Front Neuroendocrinol 20, 270-295, 1999. doi:10.1006/frne.1999.0186

Pilo B, John Tm, Pemsingh Rs, George Jc: Post-vagotomy changes in the ultrastructure of the thyroid gland and circulating levels of its hormones in the pigeon. Cytobios 41, 175-180, 1984.

Powley Tl, Phillips RJ: Musings on the wanderer: what's new in our understanding of vago-vagal reflexes? I. Morphology and topography of vagal afferents innervating the GI tract. Am J Physiol Gastrointest Liver Physiol 283, G1217-1225, 2002.

Randall Dc, Brown Dr, Mcguirt As, Thompson Gw, Armour Ja, Ardell Jl: Interactions within the intrinsic cardiac nervous system contribute to chronotropic regulation. Am J Physiol Regul Integr Comp Physiol 285, R1066-1075, 2003.

Rosas-ballina M, Ochani M, Parrish Wr, Ochani K, Harris Yt, Huston Jm, Chavan S, Tracey KJ: Splenic nerve is required for cholinergic antiinflammatory pathway control of TNF in endotoxemia. Proc Natl Acad Sci U S A $105,11008-11013,2008$. doi:10.1073/pnas.0803237105

Schafer Mk, Eiden Le, Weihe E: Cholinergic neurons and terminal fields revealed by immunohistochemistry for the vesicular acetylcholine transporter. II. The peripheral nervous system. Neuroscience 84, 361-376, 1998. doi:10.1016/S0306-4522(97)80196-0

Sternberg EM: Neural regulation of innate immunity: a coordinated nonspecific host response to pathogens. Nat Rev Immunol 6, 318-328, 2006. doi:10.1038/nri1810

Tracey KJ: Physiology and immunology of the cholinergic antiinflammatory pathway. J Clin Invest 117, 289-296, 2007. doi:10.1172/JCI30555

Van Der Zanden Ep, Boeckxstaens Ge, De Jonge Wj: The vagus nerve as a modulator of intestinal inflammation. Neurogastroenterol Motil 21, 6-17, 2009. doi:10.1111/j.1365-2982.2008.01252.x

Wetzel Gt, Brown JH: Presynaptic modulation of acetylcholine release from cardiac parasympathetic neurons. Am J Physiol 248, H33-39, 1985.

YANG M, ZHAO X, MisELIS RR: The origin of catecholaminergic nerve fibers in the subdiaphragmatic vagus nerve of rat. J Auton Nerv Syst 76, 108-117, 1999. doi:10.1016/S0165-1838(99)00014-4 